\title{
Sitios de cría y actividad de picadura de especies de Anopheles en el municipio de Cimitarra, Santander, Colombia
}

\author{
Helena Brochero 1, Paula Ximena Pareja ${ }^{1}$, Gloria Ortiz ${ }^{2}$, Víctor Alberto Olano ${ }^{1}$ \\ ${ }^{1}$ Laboratorio de Entomología, Instituto Nacional de Salud, Bogotá D.C., Colombia. \\ 2 Programa de Enfermedades Transmitidas por Vectores, Secretaría de Salud de Santander, Bucaramanga, \\ Santander.
}

Introducción. El municipio de Cimitarra aporta el $45 \%$ de los casos de malaria en el departamento de Santander. Los casos proceden del área urbana y rural, y aunque la población más vulnerable corresponde a la comprendida entre 15 y 45 años de edad, se registran casos de malaria en menores de un año.

Objetivo. Conocer sobre aspectos de la biología y comportamiento de las especies del género Anopheles presentes en el área con el fin de orientar estrategias de intervención para el control del vector.

Materiales y métodos. De octubre a diciembre de 2002 y en marzo de 2003 se realizaron capturas de mosquitos adultos y búsqueda de formas inmaduras de mosquitos Anopheles en el área.

Resultados. Se obtuvieron isofamilias a partir de 620 hembras silvestres, registrando las siguientes en orden de abundancia: Anopheles (Nyssorhynchus) triannulatus (Neiva y Pinto 1922), Anopheles (Nyssorhynchus) nuneztovari Gabaldon 1940, Anopheles (Nyssorhynchus) rangeli Gabaldon, Cova-García y López 1940, Anopheles (Anopheles) pseudopunctipennis Theobald 1901, Anopheles (Anopheles) mattogrosensis Lutz y Neiva 1911 y Anopheles (Anopheles) neomaculipalpus Curry 1933. La tasa más alta de picadura $(0,5)$ se registró para An. nuneztovari y An. triannulatus en el intradomicilio entre las 20 y 21 horas. Se inspeccionaron 42 sitios de cría, de los cuales $81 \%$ correspondió a estanques piscícolas, 9,5\% a charcos y $2,3 \%$ a tanques de cemento. De los estanques piscícolas, el $87 \%$ se ubicó alrededor de las viviendas y fueron positivos para anofelinos.

Conclusión. Este estudio ha evidenciado que en Cimitarra ocurren en simpatría especies crípticas y hermanas del género Anopheles, subgénero Nyssorhynchus.

Palabras clave: Anopheles, malaria/prevención y control, vectores de enfermedades, biología, Colombia.

Breeding places and biting activity of Anopheles species in the municipality of Cimitarra, Santander, Colombia

Introduction. Forty five percent of malaria cases in the department of Santander, Colombia originate in the municipality of Cimitarra. This locality has reported cases from the rural and urban areas. The population between 15 and 45 years of age is the most affected. Additionally, Cimitarra has registered malaria cases in children under 1 year of age.

Objective. To determine the biology and behavioral aspects of Anopheles mosquitoes from Cimitarra to more effectively orient control strategies.

Materials and methods. During October to December/2002 and March/2003, anopheline collections were undertaken. Isofamilies were obtained from 620 wild females.

Results. The following species were found in order of abundance: Anopheles (Nyssorhynchus) triannulatus (Neiva y Pinto 1922), Anopheles (Nyssorhynchus) nuneztovari Gabaldón 1940, Anopheles (Nyssorhynchus) rangeli Gabaldón, Cova-García y López 1940, Anopheles (Anopheles) pseudopunctipennis Theobald 1901, Anopheles (Anopheles) mattogrosensis Lutz y Neiva 1911 and Anopheles (Anopheles) neomaculipalpus Curry 1933. The highest biting rate (0.5) was recorded for An. nuneztovari and An. triannulatus indoors between 20-21 hours. 
Forty two breeding places were sampled, $81 \%$ were fish ponds, $9.5 \%$ puddles and $2.3 \%$ cement tanks. Eighty seven percent of fish ponds were located near dwellings, being positive for anopheline larvae.

Conclusion. This study has shown that in Cimitarra cryptic and sister species of Anopheles genus occur in sympatry, Nyssorhynchus subgenera.

Keywords: Anopheles, malaria/prevention \& control, disease vectors, biology, Colombia.

El departamento de Santander, ubicado en el valle del río Magdalena, está atravesado por la Cordillera Oriental y presenta una amplia variabilidad ecológica, registrando municipios con transmisión permanente de malaria como Barrancabermeja, Sabana de Torres, Puerto Parra, San Vicente de Chucurí, Simácota y Cimitarra. Cimitarra tiene una altitud de $158 \mathrm{msnm}$, una temperatura media de $28^{\circ} \mathrm{C}$, una precipitación media anual de 2.720 $\mathrm{mm}$ (1) y tiene una población flotante resultante de las actividades de ganadería y el conflicto armado interno. Estos aspectos permiten condiciones ecológicas y epidemiológicas aptas para la transmisión estable de malaria. De acuerdo con la Oficina de Epidemiología de la Secretaría de Salud de Santander, para una población estimada de 10.622 habitantes en el área urbana y 20.378 en el área rural, Cimitarra registró durante el año 2002 un total de 208 casos por Plasmodium vivax y seis casos por Plasmodium falciparum; en el 2003 se observó un aumento significativo en la transmisión por $P$. falciparum con 59 casos, en tanto que se presentaron 161 casos por $P$. vivax. De acuerdo con el Sistema de Vigilancia Nacional, Sivigila, durante el 2004 este municipio continuó mostrando una tendencia a la disminución de casos por $P$. vivax con 64 registros, y estabilidad en los casos por $P$. falciparum con 37 registros. Hasta la semana epidemiológica 31 de 2005 únicamente se habían registrado casos por $P$. vivax. En un estudio realizado por la Secretaría de Salud Departamental, se encontró que los casos en Cimitarra proceden tanto del área urbana como rural, siendo la población más vulnerable la

Correspondencia:

Helena Luisa Margarita Brochero,

Laboratorio de Entomología, Instituto Nacional de Salud,

Avenida Calle 26 № 51-60, Bogotá, D.C.-Colombia.

Tel. 2207700, telefax: 2200923

hbrochero@ins.gov.vo

Recibido: 13/12/05; aceptado: 10/05/06 comprendida entre los 15 y 45 años de edad con el $45,4 \%$ del total de casos, aunque también se registran casos en menores de un año.

En este municipio se han considerado Anopheles (Nyssorhynchus) darlingi Root 1926 y An. nuneztovari Gabaldon 1940 como principales vectores(2). Los registros del Servicio de Erradicación de la Malaria corresponden al municipio de Barrancabermeja, el cual incluía la zona de estudio de lo que hoy corresponde a Cimitarra. Sobre esta área se encontró además un informe técnico realizado por el grupo de enfermedades transmitidas por vectores de la Secretaría de Salud de Santander relativo a los sitios de cría de los anofelinos de Cimitarra, Santander. Este estudio, basado en la identificación de estadíos larvales, registra especies como An. rangeli y Anopheles trinkae Faran 1979, conocidas por ser especies hermanas de An. nuneztovari, con caracteres morfológicos solapados que dificultan su adecuada determinación taxonómica.

Con el propósito de ampliar el conocimiento sobre la biología de las especies de Anopheles presentes en esta área para contribuir a la definición de estrategias de control integrado y selectivo de vectores de malaria en la zona, se llevó a cabo un estudio de inventario de especies basado en la obtención de series e isofamilias entomológicas y su asociación con aspectos de su biología como la abundancia relativa, la actividad de picadura y las características de los sitios de cría.

\section{Materiales y métodos}

El estudio se llevó a cabo en por lo menos una vivienda de cada una de las siguientes localidades del municipio de Cimitarra, Santander, así: del área rural: vereda Cobaplata-EI Hueco N 06 39'38" O 745'28", vereda Parcelas N 06³2'4" O 7359'008', vereda Cascajero N 06²2'03" O 7360'03", vereda 
San Juancito N $06^{\circ} 21^{\prime} 008^{\prime \prime} \mathrm{O} 73^{\circ} 58^{\prime} 08^{\prime \prime}$. En el área urbana: Barrio 28 de abril $06^{\circ} 18^{\prime} 58^{\prime \prime}$ latitud norte y $73^{\circ} 57^{\prime} 02^{\prime \prime}$ longitud oeste (1).

Se recolectaron anofelinos atraídos a picar al hombre en el intra, peri y extradomicilio (3). Se eligió el dormitorio como el lugar de captura intradomiciliario, mientras que en el peridomicilio se seleccionó el lugar de mayor actividad de los habitantes. Se empleó trampa Shannon (4) y se realizaron búsquedas de mosquitos en reposo y, cuando fue posible, se empleó ganado vacuno como cebo animal (5). Las capturas se realizaron durante 50 minutos cada hora entre las 18 y 21 horas, una semana consecutiva cada mes, de octubre a diciembre de 2002 y en marzo de 2003. Los funcionarios responsables de la recolección de mosquitos se rotaron cada hora por los diferentes sitios de la vivienda con el propósito de evitar sesgo en las capturas. Las hembras se colocaron individualmente en frascos de plástico con algodón en el fondo, así como un rodete de papel de filtro humedecido para facilitar la ovipostura; los frascos se cubrieron con tela de tul para proporcionar la solución azucarada al 10\% y la ingestión de sangre se hizo con cobayo (6). Si no ocurría oviposición espontánea, se inducía retirando una pata y un ala del espécimen previamente adormecido con vapores de acetato de etilo y colocando a la hembra sobre un recipiente con agua (7). Una fracción de los huevos se almacenó en alcohol al $70 \%$ para la determinación taxonómica, mientras que la pata y el ala retiradas fueron montadas en lámina con bálsamo de Canadá.

Se inspeccionaron los sitios de cría más cercanos a las viviendas seleccionadas registrando las características de tamaño aproximado, distancia a la vivienda, exposición a la luz del sol, turbidez y movimiento del agua, y ubicación de la vegetación (3). Se obtuvieron isofamilias y series entomológicas de los ejemplares recolectados, preservando una fracción de los huevos, las pieles de larva y pupa y el mosquito obtenido durante la generación F1 (6). Las exuvias de cada estadio se fijaron en bálsamo de Canadá y se etiquetaron acorde con los datos de campo. Los estadios asociados de cada serie entomológica e isofamilia, incluida la genitalia de machos, se identificaron usando claves taxonómicas dicotómicas (8-13). Algunas determinaciones taxonómicas se confirmaron en el Smithsonian Institution, National Museum of Natural History, Department of Systematic Biology - Entomology, Washington, D.C. De cada especie se dejaron muestras en la colección de referencia del Laboratorio de Entomología del Instituto Nacional de Salud y en la Unidad de Entomología de la Secretaría de Salud de Santander.

La actividad de picadura se definió como el número de mosquitos capturados por especie aterrizando en el hombre durante cincuenta minutos consecutivos por cada operario. Se realizó un análisis de comparaciones de medias con base en la prueba $t$-Student.

\section{Aspectos éticos}

El protocolo de investigación para la realización de este estudio se encuentra inscrito en el Comité Técnico de Investigaciones del Instituto Nacional de Salud y cuenta con el aval del Comité de Ética del Instituto Nacional de Salud.

\section{Resultados}

Se recolectaron 620 mosquitos adultos hembra empleando 17 horas en intradomicilio, 50 horas en peridomicilio, 55 horas en extradomicilio, 47 horas con trampa Shannon, 5 horas en búsqueda de mosquitos en reposo y 4 horas con cebo animal. A partir de las hembras recolectadas se obtuvieron 325 isofamilias, siendo $A n$. (Nyssorhynchus) triannulatus (Neiva y Pinto 1922) la más abundante, seguida por An. (Nyssorhynchus) nuneztovari Gabaldon 1940, An. (Nyssorhynchus) rangeli Gabaldon, Cova García y López 1940, Anopheles (Anopheles) pseudopunctipennis Theobald 1901, Anopheles (Anopheles) mattogrosensis Lutz y Neiva 1911 y Anopheles (Anopheles) neomaculipalpus Curry 1933. La abundancia relativa de cada una de las especies con los diferentes métodos de captura y la actividad de picadura para cada especie se observan en el cuadro 1. Cuando se analizaron estas variables para todas las especies en los diferentes horarios y sitios de captura, no se evidenciaron diferencias estadísticamente significativas, por lo que se consideró para cada 
Cuadro 1. Abundancia relativa de cada una de las especies según los diferentes métodos y sitios de captura y actividad de picadura para mosquitos Anopheles capturados en el municipio de Cimitarra, Santander, Colombia 2002-2003.

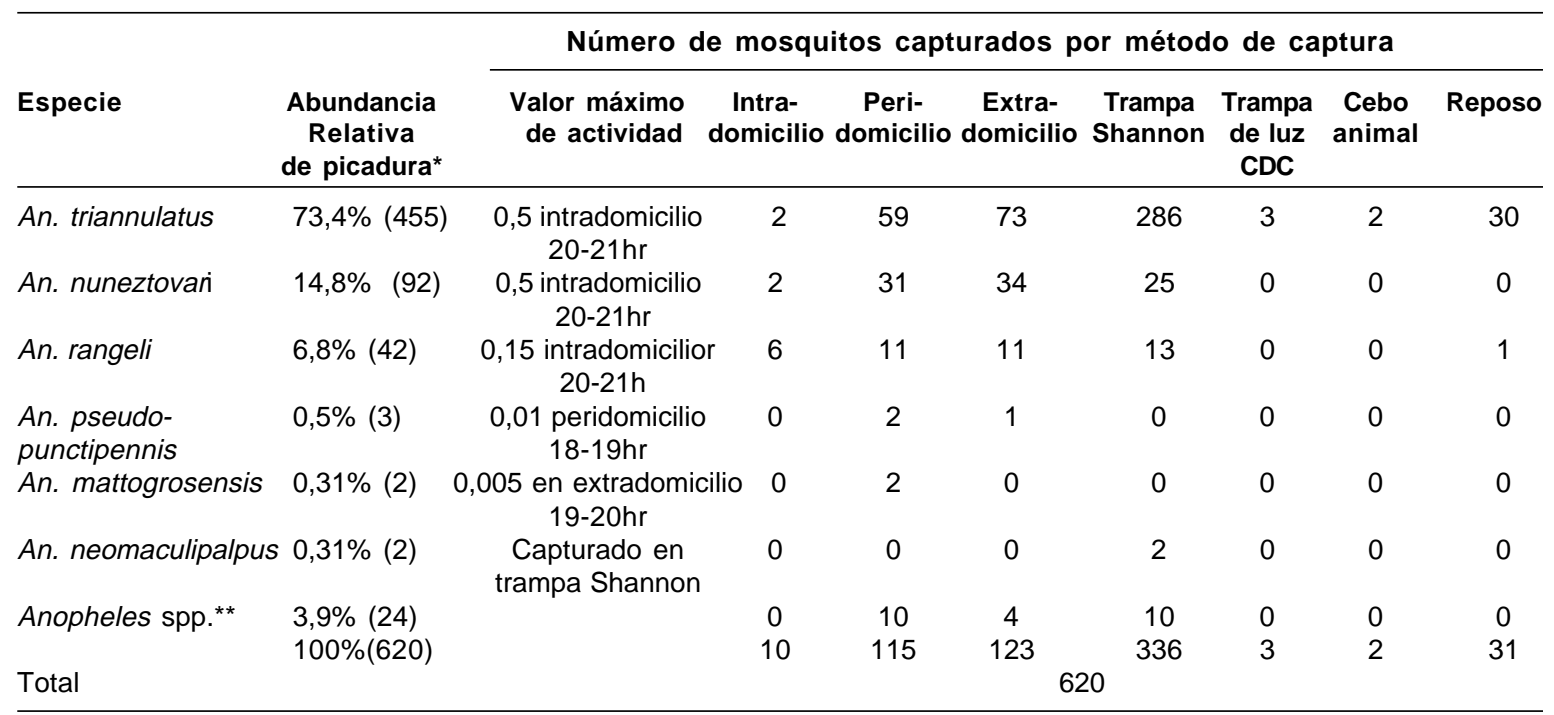

Actividad de picadura $=$ número de mosquitos capturados $/ 50$ minutos/hombre

${ }^{* *}$ No pudieron ser identificados por encontrarse en mal estado.

especie el valor promedio mayor de actividad de picadura y abundancia relativa. Cuando se analizó el comportamiento de picadura por mes de estudio se encontraron diferencias estadísticamente significativas $(\mathrm{t}=-2,92 ; p<0,0059)$, siendo diciembre el mes de mayor actividad hematofágica. También en diciembre se logró la mayor cantidad de anofelinos, hallándose $A n$. triannulatus en grandes cantidades en el intra, peri y extradomicilio, como también fuertemente atraída por la luz, ya que la mayor densidad de captura se registró en trampa Shannon.

Para An. nuneztovari la captura con atrayente humano fue la más eficaz, a pesar de haber sido capturada en menor proporción con relación a An. triannulatus (cuadro 1). Del total de mosquitos capturados, el $73 \%$ de los ejemplares de $A n$. nuneztovari y el $66 \%$ de An. rangeli se recolectaron atraídos por el hombre, pero sólo el $30 \%$ de An. triannulatus. No se registró $A n$. pseudopunctipennis dentro de las viviendas ni atraída por trampa Shannon, mientras que An. neomaculipalpus sólo se halló en trampa Shannon.

Se inspeccionaron 42 criaderos, correspondiendo el $81 \%$ a estanques piscícolas, $9,5 \%$ a charcos, $4,8 \%$ a margen de ríos y $2,3 \%$ a tanques de cemento y canales de desagüe. De los 37 sitios de cría del área rural, los estanques piscícolas representaron el $88 \%$ con un $86,7 \%$ de positividad, constituyendo An. triannulatus la especie más abundante. Los criaderos restantes del área rural correspondieron a charcos de agua lluvia (9\%) y márgenes de ríos (4\%), de los cuales sólo se encontró positivo este último con An. triannulatus. En el área urbana, el $40 \%$ correspondió a estanques piscícolas y el porcentaje restante correspondió a charcos, márgenes de río y tanques de cemento (cuadro 2). En general, los sitios de cría constituyeron criaderos con agua permanente, clara o turbia, totalmente expuestos al sol, con abundante vegetación circundante y emergente, particularmente gramíneas. La temperatura del agua hacia el medio día para los estanques piscícolas fue de $32,5^{\circ} \mathrm{C}$ (mínima $29,5^{\circ} \mathrm{C}$, máxima $39^{\circ} \mathrm{C}$ ) y el pH osciló entre 6,1 y 8,6 .

Los estanques para cría de peces representaron los criaderos más abundantes, con gran variabilidad de especies, abundante presencia de larvas y pupas, marcada cercanía a las viviendas tanto en áreas rurales como urbanas, ya que constituye un sustento económico de los 
Cuadro 2. Abundancia de anofelinos por tipo de criadero y por localidad en el municipio de Cimitarra, Santander, Colombia 2002-2003.

\begin{tabular}{|c|c|c|c|c|c|}
\hline & \multirow[t]{2}{*}{ Localidad } & \multicolumn{2}{|c|}{ Criaderos } & \multirow[b]{2}{*}{ Cantidad } & \multirow[b]{2}{*}{ Especies } \\
\hline & & Cantidad & Tipo & & \\
\hline & $\begin{array}{l}\text { Vereda } \\
\text { Cobaplata el Hueco }\end{array}$ & 1 & $\begin{array}{l}\text { Estanque piscícola } \\
\text { Charco }\end{array}$ & $\begin{array}{r}214 \\
13 \\
10 \\
1 \\
0\end{array}$ & $\begin{array}{l}\text { An. triannulatus } \\
\text { An. nuneztovari } \\
\text { An. rangeli } \\
\text { An.pseudopunctipennis }\end{array}$ \\
\hline Área & $\begin{array}{l}\text { Vereda } \\
\text { La Cascajera }\end{array}$ & 2 & Estanque piscícola & 1 & An. rangeli \\
\hline rural & $\begin{array}{l}\text { Vereda } \\
\text { Parcelas }\end{array}$ & $\begin{array}{l}6 \\
1\end{array}$ & $\begin{array}{l}\text { Estanque piscícola } \\
\text { Margen de río }\end{array}$ & $\begin{array}{r}52 \\
13 \\
18 \\
4\end{array}$ & $\begin{array}{l}\text { An. triannulatus } \\
\text { An. nuneztovari } \\
\text { An. rangeli } \\
\text { An. triannulatus }\end{array}$ \\
\hline & $\begin{array}{l}\text { Vereda } \\
\text { San Juanchito }\end{array}$ & $\begin{array}{l}5 \\
2 \\
1\end{array}$ & $\begin{array}{l}\text { Estanque piscícola } \\
\text { Charcos } \\
\text { Canal de desagüe }\end{array}$ & $\begin{array}{l}1 \\
4 \\
1 \\
0\end{array}$ & $\begin{array}{l}\text { An. triannulatus } \\
\text { An. rangeli } \\
\text { An. triannulatus }\end{array}$ \\
\hline Área urbana & $\begin{array}{l}\text { Barrio } \\
28 \text { de abril }\end{array}$ & $\begin{array}{l}1 \\
1 \\
1\end{array}$ & $\begin{array}{l}\text { Estanque piscícola } \\
\text { Charco } \\
\text { Tanque de cemento } \\
\text { Margen de río }\end{array}$ & $\begin{array}{r}19 \\
2 \\
4 \\
0 \\
0 \\
0\end{array}$ & $\begin{array}{l}\text { An. } \text { triannulatus } \\
\text { An. nuneztovari } \\
\text { An. rangeli }\end{array}$ \\
\hline
\end{tabular}

habitantes del área (cuadro 2). Algunos se encontraron abandonados, lo que hace que la vegetación aumente, permitiendo una mejor adaptación de las formas inmaduras de mosquitos.

Se registró An. nuneztovari y An. rangeli en criaderos del área urbana y rural. An. triannulatus, $A n$. nuneztovari y $A n$. rangeli se hallaron compartiendo el mismo tipo de criaderos, estanques piscícolas. An. pseudopunctipennis se encontró sólo en un estanque piscícola en el área rural.

\section{Discusión}

En Colombia, Anopheles (Nyssorhynchus) albimanus Wiedemann 1820, An. darlingi y An. nuneztovari se consideran vectores primarios de malaria, mientras que Anopheles (Anopheles) puntimacula Dyar and Knab 1906, Anopheles (Kerteszia) lepidotus Zavortink 1973, Anopheles (Kerteszia) neivai Howard, Dyar and Knab 1912 y An. pseudopunctipennis Theobald 1901 han sido señalados como vectores secundarios $(2,14,15)$. El Servicio de Erradicación de la Malaria y la
Secretaría de Salud de Santander registran como vectores para Cimitarra a An. nuneztovari, An. pseudopunctipennis y An. darlingi, este último restringido a la vereda Guayabito (2). An. nuneztovari constituye un complejo de especies conformado por formas crípticas con diferentes patrones en el comportamiento de picadura y características citogenéticas particulares (16-20). Esta especie está relacionada filogenéticamente con An. rangeli y Anopheles (Nyssorhynchus) trinkae Faran 1979, presentando similitud morfológica interespecífica y alta variabilidad intraespecfíca (21-23). Estos aspectos hacen difícil la adecuada determinación taxonómica de especies cuando se hallan en simpatría. Basados en la caracterización morfológica de todos los estadios asociados de isofamilias, incluida la genitalia de machos y la secuenciación del fragmento ITS2 del ADN ribosomal de mosquitos capturados en las localidades de estudio, se pudo confirmar el registro de Anopheles nuneztovari para el municipio de Cimitarra (datos de secuencia ADNr no mostrados). Se han documentado 
diferentes patrones de picadura para esta especie. Una población se caracteriza por tener hábitos exofílicos, pica al hombre hacia la puesta del sol, entre las 18:00 y 19:00 horas, y puede actuar como vector auxiliar a nivel regional $(20,24)$; otra es zoofílica, crepuscular y peridomiciliar $(25,26)$, y una tercera pica al hombre dentro de las casas, tarde en la noche, y actúa como vector primario de malaria $(27,28)$. Aunque en este estudio sólo se efectuaron capturas entre las 18 y las 21 horas debido a dificultades propias del conflicto armado en la zona, se pudo observar que la tendencia de An. nuneztovari en Cimitarra corresponde a este último patrón. An. nuneztovari ha sido detectada en el norte de Colombia y el occidente de Venezuela como vector principal de malaria, correspondiendo probablemente al citotipo $\mathrm{B}$.

An. nuneztovari presenta gran adaptabilidad a la colonización de criaderos artificiales y, en general, a cambios bruscos producidos por el hombre al medioambiente (Olano V, Brochero H, Córdoba F, Arévalo C. New breeding places for Anopheles in Colombia. Abstract 68th Annual Meeting of the American Mosquito Control Association and the West Central Mosquito \& Vector Control Association. February 16-21 de 2002. Denver, Colorado, USA) $(26,29,30)$. En Cimitarra, esta especie se encontró bien adaptada a criaderos permanentes hechos por el hombre y ubicados cerca de las viviendas, totalmente expuestos al sol, con vegetación circundante y emergente, los cuales constituyeron el $81 \%$ de los criaderos inspeccionados.

Nuestro estudio no registró An. darlingi. Se ha descrito cómo esta especie prefiere criaderos sombreados, de aguas limpias con corriente lenta (31). El no haber capturado especímenes de esta especie en los sitios estudiados en Cimitarra se podría explicar por el hecho de que los criaderos más abundantes y de mayor variabilidad de especies fueron los estanques de cría de peces. Tadei y Tacher encontraron que al inicio de la construcción de una autopista, An darlingi coexistía con An. nuneztovari en altas densidades; posteriormente se observó un incremento considerable en las densidades de An. nuneztovari y una reducción en An darlingi, justificada por la deforestación que incrementaba la distancia entre el final del bosque y los dormitorios de las personas, provocando también que los criaderos de los mosquitos quedaran expuestos al sol, condición ésta óptima para An. nuneztovari, aunque menos apta para An. darlingi (32). Por las características de su economía, la ecología de Cimitarra ha sufrido cambios importantes determinados por la tala de árboles para actividades de ganadería, monocultivos y excavación de tierras cercanas a las casas para formar estanques de cría de peces, aspectos de fuerte presión antrópica que pueden explicar que An. darlingi se halle restringida a un área del municipio mientras que $A$. nuneztovari constituya una especie abundante. Dada su marcada tendencia antropofílica, su endofilia, con una actividad de picadura máxima en el intradomicilio $(0,5)$, su abundancia en la zona y al hecho de que ha sido registrada para el país naturalmente infectada por $P$. vivax y por $P$. falciparum (32), $A n$. nuneztovari podría estar actuando como vector primario de malaria en Cimitarra.

En este estudio, An. triannulatus constituyó la especie más abundante, hallándose una correlación positiva entre la abundancia registrada en criaderos y la obtenida para adultos en una misma zona. Se ha descrito que An. triannulatus presenta una actividad de picadura crepuscular, momento en que la gente está frecuentemente activa en el peridomicilio; ha sido hallada naturalmente infectada con $P$. vivax y $P$. falciparum (22,33-35), lo que aunado a su abundancia (36-39), su tendencia endofágica (Quiñones M, Linton Y, Harbach R, Estrada D, Erazo H, Calle D, et al. Malaria vector species in southern Colombia: species determination and natural infectivity. XVth International Congress for Tropical Medicine and Malaria. Cartagena de Indias, Colombia, August 20-25, 2000:108), su adaptabilidad a cambios bruscos del medio producidos por el hombre y su oportunismo para alimentarse de humanos (32), se ha considerado como vector regional auxiliar en Brasil (36-40). En Cimitarra, esta especie presentó una actividad de picadura intradomiciliaria igual a la registrada para An. nuneztovari cuando los habitantes de la zona se encuentran durmiendo dentro de las viviendas, particularmente en el área rural. 
An. rangeli no se considera vector de malaria en Colombia, pero algunos estudios en el sur del país han detectado alta infectividad por Plasmodia humano $\mathrm{y}$, ante la ausencia de otras especies consideradas como vectores primarios de malaria, se le ha incriminado como posible vector en el departamento de Putumayo, Colombia (39). Se ha registrado tendencia endofílica (41) y adaptación a cambios producidos por el hombre en esta especie, por lo que en Cimitarra, donde presentó una tasa de picadura al interior de los dormitorios igual a la de $A n$. nuneztovari y $A n$. triannulatus, compartiendo hábitat con éstas y constituyendo las especies más abundantes, se hace necesario mantener la vigilancia entomológica para esta especie.

Aunque An. neomaculipalpus se ha hallado naturalmente infectado por $P$. falciparum (14) en el país, en Cimitarra no se consideró de importancia en salud pública debido a que se encontró en bajas densidades durante todo el estudio y con poca tendencia antropofílica, ya que sólo fue recolectado mediante trampa Shannon ubicada en el extradomicilio.

Lounibos y Conn, 2000, indican que los eventos de malaria en Sur América ocurren por la presencia de un vector de amplia distribución, pero se mantienen por la presencia de vectores regionales (40). Dada la diversidad de especies registradas para Cimitarra, es probable que varias de ellas se encuentren incriminadas en la transmisión permanente de malaria en esta zona. Dicha heterogeneidad incidiría de manera importante en la epidemiología de la enfermedad, ya que estas especies pueden diferir en su capacidad para desarrollar parásitos Plasmodia, en su actividad de picadura, que fluctúa de acuerdo con las densidades poblacionales y con la estacionalidad local, estos aspectos deben tenerse en cuenta para la implementación de programas de prevención y control, particularmente de toldillos impregnados con insecticidas.

Este estudio ha evidenciado que en Cimitarra ocurren en simpatría especies crípticas y hermanas del género Anopheles, subgénero Nyssorhynchus, de tal manera que en actividades de vigilancia entomológica regular y durante brotes o epidemias por malaria en la zona, se hace necesaria la obtención de series entomológicas e isofamilias a partir del material entomológico recolectado que permita una adecuada determinación taxonómica y, por ende, una correcta asociación con aspectos de la biología, la actividad de picadura y la infectividad con parásitos Plasmodia humano para cada especie. En este municipio ocurre transmisión de malaria en el intradomicilio afectando a menores de un año, por lo que se hace necesario efectuar estudios durante toda la noche que permitan complementar los datos sobre comportamiento de picadura y determinar la validez de los toldillos impregnados con insecticidas como medida de elección para el control vectorial. Se sugiere desarrollar estrategias de participación y acción comunitaria para el desbroce de la vegetación circundante de los estanques activos para cría de peces, lo que permitiría la depredación de larvas, y el relleno de estanques abandonados (22), debido a que fueron los más abundantes y aptos para el desarrollo de An. nuneztovari, considerado vector primario de malaria en Colombia

\section{Agradecimientos}

A Richard Wilkerson, director de Biosystematic Unit, Walter Reed, National Museum of Natural History - Smithsonian Institution, Washington D.C., por la confirmación en la identificación taxonómica. A la Secretaría de Salud de Santander por el apoyo logístico y de personal auxiliar.

\section{Conflicto de intereses}

Los autores declaran que no existe conflicto de intereses entre el Instituto Nacional de Salud, la Secretaría Departamental de Salud de Santander y la Secretaría Municipal de Salud de Cimitarra, donde se informaron los resultados del presente estudio.

\section{Financiación}

Este proyecto fue financiado por la Organización Panamericana de la Salud (Código HDP/HDR/ RGP/COL-3053), el Instituto Nacional de Salud de Colombia y la Secretaría de Salud de Santander. 


\section{Referencias}

1. Instituto Geográfico Agustín Codazzi. Diccionario geográfico de Colombia. Tomo IV. Santa Fé de Bogotá: Reprolaser LTDA; 1996. p.2459-60.

2. Servicio de Erradicación de la Malaria. Plan de Erradicación de la Malaria en Colombia. Volumen I y II. Bogotá: Ministerio de Salud Nacional; 1957.

3. World Health Organization. Manual of practical entomology in malaria. Vol II. Methods and techniques. Geneva: WHO; 1975. p.13.

4. Service M. A critical review of procedures for sampling populations of adult mosquitoes. Bull Entomol Res 1977;67:343-82.

5. Fleming G. Biología y ecología de los vectores de la malaria en las Américas. Washington D.C.: Organización Panamericana de la Salud; 1986. p.13-6.

6. Belkin J, Schick R, Galindo P, Aitken T. Estudios sobre mosquitos (Diptera: Culicidae). la. Un proyecto para un estudio sistemático de los mosquitos de mesoamérica. Ila. Métodos para coleccionar, criar y preservar mosquitos. Contrib Am Entomol Inst 1967;1:163-80.

7. Estrada DA, Quiñones ML, Sierra DM, Calle DA, Ruiz F, Erazo HF, et al. Utilidad de la morfología de los huevos como método indirecto para identificar $A n$ benarrochi, An oswaldoi y An rangeli en Putumayo, Colombia. Biomédica 2003;23:388-95.

8. Rubio-Palis Y. Anopheles (Nyssorhynchus) de Venezuela: taxonomía, bionomía, ecología e importancia médica. Maracay: Escuela de Malariologia y Saneamiento Ambiental; 2000. p.120.

9. Faran ME. Mosquito studies (Diptera: Culicidae) XXXIV A revision of the Albimanus Section of the subgenus Nyssorhynchus of Anopheles. Contrib Am Entomol Inst 1980;15:1-215.

10. Faran ME, Linthicum KJ. A handbook of the Amazonian species of Anopheles (Nyssorhynchus) (Diptera: Culicidae). Mosq Syst 1981;13:1-81.

11. Linthicum KJ. A revisión of the Argyritarsis Section of the subgenus Nyssorhynchus of Anopheles. Mosq Syst 1988;20:98-271.

12. Linley JR, Lounibos LP, Conn J, Duzak D, Nishimura N. A description and morphometric comparison of eggs form eight geographic populations of the South American malaria vector $A n$. (Nyssorhynchus) nunez-tovari (Diptera: Culicidae). J Am Mosq Control Assoc 1996;12:275-92.

13. Lounibos LP, Duzak D, Linley JR. Comparative egg morphology of six species of the Albimanus Section of Anopheles (Nyssorhynchus) (Diptera: Culicidae). J Med Entomol 1997;34:136-55.

14. Herrera S, Suárez M, Sánchez G, Quiñones M. Uso de la técnica inmunorradiométrica (IRMA) en anofelinos de Colombia para la identificación de esporozoitos de Plasmodium. Colomb Med 1987;18:2-6.

15. Olano V, Brochero H, Sáenz R, Quiñones M, Molina J. Mapas preliminares de la distribución de Anopheles vectores de malaria en Colombia. Biomédica $2001 ; 21: 402-8$.

16. Conn J, Puertas YR, Seawright JA. A new cytotype of An nuneztovari form western Venezuela and Colombia. Am Mosq Control Assoc 1993;9:294-301.

17. Conn J. A genetic study of the malaria vector Anopheles nuneztovari from western Venezuela. J Am Mosq Control Assoc 1990;6:400-5.

18. Olano V, Carrasquilla G, Méndez F. Transmisión de la malaria urbana en Buenaventura, Colombia: aspectos entomológicos. Rev Panam Salud Publica 1997;1:28794

19. Rosa-Freitas MG, Lourenço-de-Oliveira R, de Carvalho-Pinto JC, Flores-Mendoza C, Silva-doNacimento TF. Anopheline species complexes in Brazil. Current knowledge of those related to malaria transmission. Mem Inst Oswaldo Cruz 1998;93:651-5.

20. Elliot R. Studies on man vector contact in some malarious areas in Colombia. Bull Bull World Health Organ 1968;38:239-53.

21. Hribar L. Geographic variation of male genitalia of Anopheles nuneztovari (Diptera: Culicidae). Mosq Syst 1994;26:132-44

22. Hribar L. Costal wing spot variation within and among progeny of single female An. nuneztovari (Diptera: Culicidae). Mosq Syst 1995;27:1-15

23. Fritz G, Conn J, Cockburn F, Seawrigth J. Sequence analysis of the rDNA internal transcribed spacer 2 form geographic strains of An nuneztovari. (Diptera: Culicidae). Mol Biol Evol 1994;11:406-16.

24. Panday RS. Anopheles nuneztovari and malaria transmission in Surinam. Mosq News 1977; 37:72837.

25. Tadei P, Corrala J. Biología de Anofelinos amazónicos IV. Observaciones sobre a actividade do picar de $A n$ nuneztovari. Acta Amazonica 1982;12:71-4.

26. Tadei $\mathbf{P}$, Dutary Thatcher $\mathbf{B}$. Malaria vectors in the Brazilian amazon: Anopheles of the subgenus Nyssorhynchus. Rev Inst Med Trop Sao Paulo 2000;42:87-94.

27. Elliot R. The influence of vector behavior on malaria transmission. Am J Trop Med Hyg 1972; 21:755-63.

28. Gabaldon A. Anopheles nuneztovari: importante vector y agente de malaria refractaria en Venezuela. Bol Dir Malar Saneam Amb 1981;21:28-38.

29. Lourenco de Oliveira R, Guimaraes AE, Arle M, Silva TF, Castro MG, Motta MA, et al. Anopheline species some of their habits and relation to malaria in endemic 
areas of Rondonia State, Amazon Region of Brazil. Mem Inst Oswaldo Cruz 1989;84:501-14.

30. Degallier N, Travassos da Rosa A, Vasconcelos $\mathbf{P}$, Hevre J, Filho Sa C, Travassos da Rosa J, et al. Modifications of arbovirus transmision in relation to construction of dams in Brazilian Amazonia. Ciencia e Cultura 1992;44:124-35

31. Brochero HL, Rey G, Buitrago LS, Olano VA. Biting activity and breeding sites of Anopheles species in the municipality of Villavicencio, Meta, Colombia. J Am Mosq Control Assoc 2005;21:182-6.

32. Rey H, Renjifo S. An nuneztovari infestado en la naturaleza con Plasmodium sp. Cespedesia 1974;3:255-61.

33. Rebelo JM, da Silva AR, Ferreira LA, Vieira JA. Anopheles (Culicidae, Anophelinae) and Malaria in Buriticupu-Santa Luzia, pre-Amazonic Maranhao. Rev Soc Bras Med Trop 1997; 30:107-11.

34. Klein TA, Lima JB, Tada MS, Miller R. Comparative susceptibility of anopheline mosquitoes in Rondonia, Brazil to infection by Plasmodium vivax. Am J Trop Med Hyg 1991;45:463-70.

35. Lanelli RV, Honorio NA, Lima DC, Lourenco-DeOliveira R, Santos RV, Coimbra Junior CE. Faunal composition and behavior of anopheline mosquitoes in the Xavante Indian reservation of Pimentel Barbosa, central Brazil. Parasite 1998;5:197-202.

36. de Arruda M, Carvalho MB, Nussenzweig RS, Maracic M, Ferreira AW, Cochrane AH. Potential vectors of malaria and their different susceptibility to Plasmodium falciparum and Plasmodium vivax in Northern Brazil identified by immunoassay. Am J Trop Med Hyg 1986;35:873-81.

37. Deane LM, Daniel Ribeiro C, Lourenco de Oliveira R, Oliveira-Ferreira J, Guimaraes A. Study on the natural history of malaria in areas of Rondonia State, Brazil and the problems related to its control. Rev Ins Med Trop Sao Paulo 1988;30:153-6.

38. Teodoro U, Guilherme AL, Lozovei AL, La Salvia Filho V, Fukushigue Y, Spinosa RP, et al. Culicidae of Itaipu lake, in the Parana river, southern Brazil. Rev Saude Publica 1995;29:6-14.

39. Quiñones M, Suárez M, Fleming F. Distribución y bionomía de los anofelinos de la costa Pacífica colombiana. Colomb Med 1987;18:19-24.

40. Lounibos LP, Conn JE. Malaria vector heterogeneity in South America. American Entomologist 2000;46:238-49.

41. Rubio-Palis Y. Abundancia y actividad hematofágica de Anopheles rangeli, An strodei y An neomaculipalpus. Bol Dir Malar Saneam Amb 1992;32:59-67. 